\title{
Health technology assessment (HTA) increasingly important in spine research
}

\author{
Maurits van Tulder
}

Received: 9 June 2011/Published online: 1 July 2011

(C) The Author(s) 2011. This article is published with open access at Springerlink.com

The European Spine Journal acknowledges the increasing attention for economic evaluations and would like to stimulate researchers to submit original papers of economic evaluations of orthopedic devices/instruments and surgical interventions for spine problems, but also pharmaceutical and conservative interventions for back and neck pain. Because back and neck pain are among the most prevalent health problems and are associated with huge costs of health care utilization and productivity losses, economic evaluations in this field are direly needed to facilitate wellinformed clinical and policy decisions.

Health care systems in western countries are becoming more expensive every year. OECD health data from 2010 show that most western countries spend about $8-16 \%$ of their gross national product on health care. Although this percentage will even grow in the near future, we cannot afford to reimburse all available health care interventions. Consequently, policy makers have to make decisions about inclusion (or exclusion) of interventions in public health insurance systems.

To be able to make well-informed decisions, it is of utmost importance that policy makers have valid and reliable information available about safety, effectiveness and cost effectiveness of interventions. Health technology assessment (HTA) is a reasonably new field of scientific research that involves evaluation of health interventions (technologies). These can be preventive, diagnostic or therapeutic interventions and either new or existing interventions. Some overlap exists between HTA and public

M. van Tulder $(\square)$

Department of Health Sciences and EMGO, Institute for Health and Care Research, VU University Amsterdam, $1081 \mathrm{HV}$ Amsterdam, The Netherlands

e-mail: maurits.van.tulder@vu.nl health research and (clinical) epidemiology, as these disciplines also evaluate effectiveness of interventions. However, besides effectiveness HTA also looks at other aspects, such as costs, side effects, organizational, legal or ethical aspects.

Ideally, interventions should be evaluated before they are widely used, because it is really difficult to withdraw interventions from the health care system. In many countries, regulations for new pharmaceutical interventions are strict, but none exist for medical devices or non-pharmaceutical interventions. New drugs are only reimbursed in public health insurance systems if they are proven to be safe, effective, and cost effective compared to existing drugs for the same disease or disorder. However, new physical therapies, new exercise therapies or new surgical techniques can be introduced without first having been scrutinized in scientific research. I would argue that it is time to also start evaluating effectiveness and cost effectiveness of new surgical and non-pharmacological interventions before they are widely used.

Economic evaluations are systematic comparisons of two or more health technologies, services or programs in terms of both costs and consequences. This simultaneous comparison of the costs and consequences provides insight into which intervention is worth doing over another or whether the additional costs of an intervention are worth the effects. Economic evaluations do not necessarily answer the question what the cheapest intervention is. If an intervention is more effective than another intervention but associated with higher costs, the intervention may still be cost effective.

During the last decade, the number of economic evaluations that have been conducted and published has increased. Similar to other types of research, it is of utmost importance that economic evaluations are conducted 
according to the state-of-the-art and that their results are valid and reliable. Knowledge of the methodology of economic evaluation is necessary.

Four types of economic evaluations exist, i.e., cost effectiveness, cost utility, cost benefit, and cost minimization analysis. The principles of these types of economic evaluations are similar; the difference is the way in which the outcomes are expressed. Cost-effectiveness and costutility analyses are the most commonly used. In costeffectiveness analysis, outcomes are disease specific, and in cost-utility analysis outcomes are patients' preferences, which are expressed as quality-adjusted life-years (QALYs) or disability-adjusted life-years. The methods of economic evaluations include choosing the alternatives; identifying the perspective; identifying, measuring and valuing costs; identifying, measuring and valuing effects; and analyzing the data.

Usually economic evaluations are performed from a societal perspective taking all costs and consequences into account. However, sometimes another perspective is chosen, e.g., that of the national health care system (NHS in the UK), or that of the company or employer. The latter is relevant for economic evaluations of interventions in an occupational setting. The perspective chosen defines which costs and outcomes are considered in the economic evaluation and whether results can be generalized to other settings.

Economic evaluations always compare two or more alternatives. The best alternative is 'usual care' or the best or most widely used alternative, since these are the most informative comparisons to policy makers. If a new intervention is cost effective compared with usual care, implementation of this intervention is useful.

Costs are usually classified as direct and indirect costs, within and outside the healthcare sector. Direct costs within the healthcare sector include all costs of healthcare utilization. These costs include, e.g., costs of general practitioner care, medication, physical therapy, surgery and hospitalization.

Direct costs outside the health care sector include costs of complementary and alternative care and patients' out-ofpocket costs. Indirect costs outside the healthcare sector include costs of productivity loss, i.e., costs of absenteeism (loss of productivity while off work) and presenteeism (loss of productivity while at work). Especially in the field of spine research, these costs are often much higher than the direct costs.

Costs are often not measured directly, but through health care utilization or resource use. When all relevant resource data have been collected, the next step is valuing the costs. Costs are resource use multiplied with valid unit prices.

Analyzing and interpreting data from economic evaluations are challenging, because cost data are typically skewed. Often there are only a few subjects with high health care utilization or long periods of work absenteeism, and therefore a relatively small number of patients with high costs. Non-parametric analysis is used to deal with skewed data and bootstrapping is a method commonly used in economic evaluations. The outcome of economic evaluations is an incremental cost-effectiveness ratio (ICER), which is estimated for each outcome. The ICER is the ratio of the difference in costs of the intervention and its alternative divided by the difference in effects and indicates the additional investments needed to gain one extra unit of effect. Uncertainty of the ICER is expressed on a so-called cost-effectiveness plane, which shows the bootstrap estimates. The $x$-axis represents the difference in effects and the $y$-axis represents the difference in costs. Cost-effectiveness acceptability curves help interpreting results of economic evaluations. They show the probability that an intervention is cost effective for a wide range of threshold ratios that policymakers would be willing to pay.

As stated before, healthcare expenditures are rising, budgets are getting tighter, and choices have to be made about providing or reimbursing healthcare interventions. These choices should be made based on available evidence regarding safety, effectiveness and cost effectiveness. Information on safety should come from observational studies, information on effectiveness from randomized controlled trials and information on cost effectiveness from economic evaluations. These studies should have adequate sample sizes and be well designed so that their results are precise, valid and reliable and lead to well-informed clinical and policy decisions.

Open Access This article is distributed under the terms of the Creative Commons Attribution Noncommercial License which permits any noncommercial use, distribution, and reproduction in any medium, provided the original author(s) and source are credited. 